\title{
Micro-mechanical investigation of the effect of fine content on mechanical behavior of gap graded granular materials using DEM
}

\author{
Habib Taha ${ }^{1,2, \star}$, Ngoc-Son Nguyen ${ }^{1, \star \star}$, Didier Marot ${ }^{1}$, Abbas Hijazi $^{2}$, and Khalil Abou-Saleh ${ }^{2}$ \\ ${ }^{1}$ GeM Institute, University of Nantes, 58 rue Michel Ange, BP 420, 44606, Saint-Nazaire Cedex, France \\ ${ }^{2}$ MPLAB-Multisciplinary Physics Laboratory, Faculty of Sciences, Lebanese University, Hadat-Baabda, Lebanon
}

\begin{abstract}
In this paper, we present a micro-mechanical study of the effect of fine content on the behavior of gap graded granular samples by using numerical simulations performed with the Discrete Element Method. Different samples with fine content varied from $0 \%$ to $30 \%$ are simulated. The role of fine content in reinforcing the granular skeleton and in supporting the external deviatoric stress is then brought into the light.
\end{abstract}

\section{Introduction}

Granular materials are often used for construction of hydraulic structures such as dikes, levees, dams, etc. Under the action of the fluid flow, gap-graded or widely graded granular materials with an upwardly concave distribution are susceptible to internal erosion, during which fine particles can be detached and transported with the fluid flow through the pore space between coarse particles [1]. The loss of fine particles can impact the mechanical behavior of eroded materials, and then the stability of hydraulic structures. To predict this impact, it is important to deeply understand the effect of the fine content on the mechanical behavior of granular materials. The effect of fine content on the behavior of silty sands has been experimentally investigated by performing triaxial tests under drained conditions [2, 3] or undrained conditions [3, 4]. It was observed that fine content greatly influences the shear strength and the dilatancy of silty sands. Under undrained conditions, Thevanayagam et al. [4] found a threshold value $f_{c, t h}$ for fine content $f_{c}$. When $f_{c}<f_{c, t h}$, the collapse potential increases with an increase in fine content. The trend is opposite when $f_{c}>f_{c, t h}$. From a micromechanical point of view, the dependence of macroscopic properties of widely graded materials on fine content can be attributed to a modification of their micro-structure. According to Thevanayagam et al. [4], for fine content $f_{c}<f_{c, t h}$, contacts between coarse grains play the primary role in the micro-structure, while for $f_{c}>f_{c, t h}$, contacts between fine grains are dominant. However, this is just a conjecture without any experimental verification. It is not clear yet how fine content reinforces granular skeleton and participates in supporting the external loading. It is worth mentioning that it is difficult to investigate experimentally the granular micro-structure. To overcome this

\footnotetext{
^e-mail: habib.taha@etu.univ-nantes.fr

${ }^{\star}$ e-mail: ngocson.nguyen@univ-nantes.fr
}

difficulty, numerical simulation of granular samples using the Discrete Element Method (DEM) can be performed to investigate the granular micro-structure.

In this paper, we use the DEM to simulate triaxial loading tests on gap graded granular samples with different fine contents. Firstly, simulated granular samples are presented and their macroscopic behavior is analyzed. The effect of fine content on the micro-structure is then investigated.

\section{Simulated samples}

YADE, an open-source framework for the DEM [5], is used for numerical simulations. Particles are spherical and the particle size distribution follows the idealized gapgraded curve shown in Figure 1. Each sample is composed of a coarse content and a fine content $f_{c}$ which varies from $0 \%$ to $30 \%$ with a step of $5 \%$. The gap ratio $G_{r}=D_{\min } / d_{\max }$ is defined as the ratio of the minimum diameter for the coarse content to the maximum diameter for the fine content. According to Chang and Zhang [6], internal instability due to seepage flow for a gap graded material might occur for a gap ratio $G_{r} \geq 3.0$. In our simulations, the gap ratio $G_{r}=3$ is chosen. The number of coarse particles is chosen depending on fine content $f_{c}$ such that the computation time is reasonable and the representativity of simulated samples is guaranteed.

The interaction between two particles $i$ and $j$ is described by two linear springs with stiffnesses $k_{n}$ and $k_{s}$ in the normal and tangential directions, respectively. The normal stiffness $k_{n}$ is defined as $k_{n}=\left(k_{i} k_{j}\right) /\left(k_{i}+k_{j}\right)$ with $k_{i}$ is the stiffness of particle $i$. In our simulations, we chose $k_{i} / d_{i}=250 \mathrm{MPa}\left(d_{i}\right.$ is the diameter of particle $\left.i\right)$ and $k_{s}=0.5 k_{n}$. The tangential interaction respects Coulomb's friction law with friction angle $\varphi=35^{\circ}$.

Particles are first generated into a cube composed of six rigid walls. At this stage, each particle diameter is reduced by a factor of 2.0. Particles are then progressively 


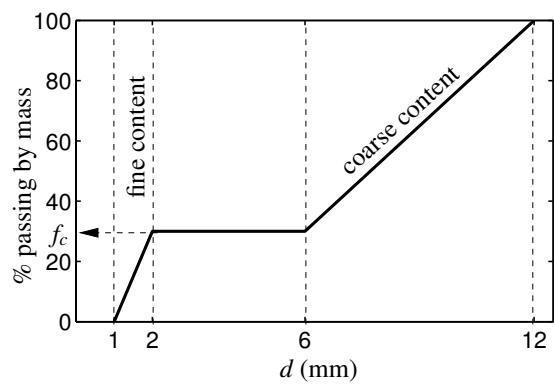

Figure 1. Gap-graded size distribution curve for the simulated samples.

expanded to reach the target size distribution. After that, the box dimensions are slowly reduced until the stresses $\sigma_{i}, i=1,2,3$ reach the confinement stress of $100 \mathrm{kPa}$. During the compaction process, the friction between particles is set to 0 to obtain dense samples. At the end of this process, the friction is reset to its original value.

The density of a fines-coarses mixture can be described by the classical void ratio, $e$, and the apparent intergranular and interfine void ratios, $e_{c}$ and $e_{f}$ defined in Equation (1). The intergranular void ratio $e_{c}$ is defined by considering that the fine grains are inactive so they are a part of voids between coarse grains. The interfine void ratio $e_{f}$ is defined as the ratio of the effective volume of interfine voids to the solid volume of fine grains.

$$
e_{c}=\frac{e+f_{c} / 100}{1-f_{c} / 100}, \quad e_{f}=\frac{e}{f_{c} / 100}
$$

Figure 2 shows the void ratios $e, e_{c}$ and $e_{f}$ versus fine content $f_{c}$ : the values of $e$ and $e_{c}$ are represented in the left axis, while the values of $e_{f}$ is represented in the right axis. Fine grains fill voids between coarse grains so the classical void ratio $e$ decreases with $f_{c}$. Lade et al. [7] experimentally found that the void ratio of sands decreases with fine content when $f_{c}<40 \%$. We can also see that the intergranular void ratio $e_{c}$ increases with $f_{c}$ since fine grains tend to intercalate between coarse grains. On the other hand, the fine fraction becomes denser with increasing fine content.



Figure 2. Void ratios $e, e_{c}$ and $e_{f}$ versus fine content $f_{c}$

\section{Triaxial compression tests}

Triaxial compression tests are performed on numerical samples. Figure 3 shows the stress ratio $q / p$ (deviator stress $q=\sigma_{1}-\sigma_{3}$ and mean stress $p=\left(\sigma_{1}+\sigma_{2}+\sigma_{3}\right) / 3$ and volumetric strain $\varepsilon_{\mathrm{v}}$ versus axial strain $\varepsilon_{11}$ for different fine contents. Stress ratio $q / p$ at the peak and critical states are shown in Table 1. It is shown that the effect of fine content on the macroscopic behavior is not significant for $f_{c}<20 \%$. However, for $f_{c} \geq 20 \%$, the shear strength and the dilatancy increases with $f_{c}$ and we can see a marked softening behavior .
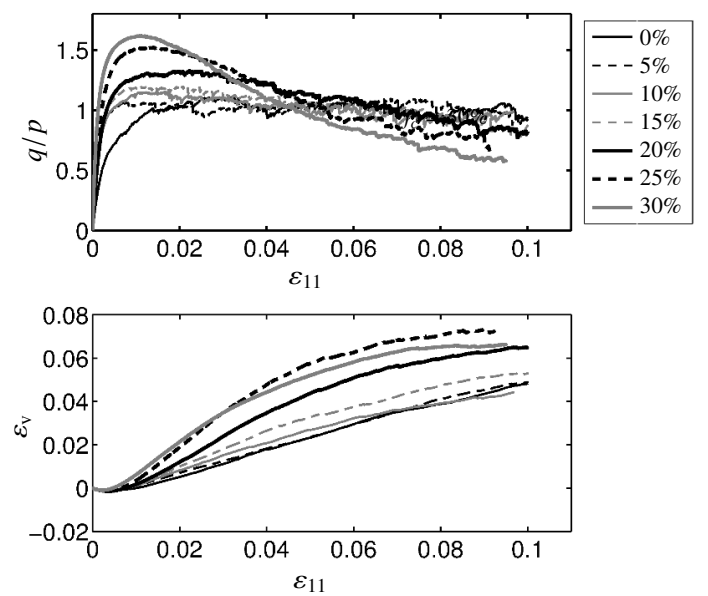

Figure 3. Stress ratio $q / p$ and volumetric strain $\varepsilon_{\mathrm{v}}$ versus axial strain $\varepsilon_{11}$ for different fine contents $f_{c}$.

Table 1. Stress ratios $(q / p)_{\text {peak }}$ and $(q / p)_{\text {critical }}$ at the peak and critical states for different fine contents $f_{c}$.

\begin{tabular}{llllllll}
\hline$f_{c}(\%)$ & 0 & 5 & 10 & 15 & 20 & 25 & 30 \\
\hline$(q / p)_{\text {peak }}$ & 1.1 & 1.1 & 1.2 & 1.2 & 1.3 & 1.5 & 1.6 \\
\hline$(q / p)_{\text {critical }}$ & 0.9 & 0.9 & 1.0 & 0.9 & 0.8 & 0.7 & 0.6 \\
\hline
\end{tabular}

\section{Microscopic investigation}

Contacts between particles in a gap graded sample can be split into three categories: coarse-coarse $(C-C)$, coarsefine $(C-F)$ and fine-fine $(F-F)$ contacts. In the following, we analyze the effect of fine content on coordination numbers defined for each category of contacts, on the anisotropy and on the stress transmission.

\subsection{Coordination numbers}

The coordination number, which is defined as the average number of contacts per particle, is often used to describe the density of a granular sample at the micro-scale. This definition seems to be not appropriate for a gap-graded sample for which the number of contacts on each coarse grain might be much higher than on each fine grain. For such a system, a coordination number for coarse fraction should be separated from the one defined for fine fraction as suggested in [8]. In this study, we define three coordination numbers $\mathcal{N}_{C}^{C-C}, \mathcal{N}_{C}^{C-F}$ and $\mathcal{N}_{F}$ which correspond to the respective average numbers of $C-C$ contacts per coarse particle, of $C-F$ contacts per coarse particle and of contacts (both $C-F$ and $F-F$ ) per fine particle.

Figure 4 shows $\mathcal{N}_{C}^{C-C}, \mathcal{N}_{C}^{C-F}$ and $\mathcal{N}_{F}$ versus fine content $f_{c}$ at the initial, peak and critical states. It can be seen that for $f_{c}<20 \%$, coarse particles constitute the primary 
skeleton with $\mathcal{N}_{C}^{C-C}$ dominant with respect to $\mathcal{N}_{C}^{C-F}$ and $\mathcal{N}_{F}$. This is one of the main reasons which explain why the macroscopic behavior is not significantly affected by fine content $f_{c}<20 \%$ as shown in Figure 3. For $f_{c} \geq 20 \%$, fine particles come into contact with coarse particles, leading to a quick increase of $\mathcal{N}_{C}^{C-F}$ with $f_{c}$. For the sample with $f_{c}=30 \%$ at the initial state, there is on average 45 fine particles around a coarse particle. Shire et al. [9] obtained a similar result for their simulations with higher gap ratio $G_{r}$. Note that $\mathcal{N}_{C}^{C-C}$ at the initial state is not affected by $f_{c} \leq 30 \%$, meaning that the initial primary skeleton formed by coarse particles is not altered by the fine fraction: fine particles actually surround and reinforce this primary skeleton.

The reinforcement of the primary skeleton by fine particles gives the ability to granular samples to dilate better so they can resist better the shear loading. On the other hand, the dilatancy leads to a loss of inter-particle contacts, and hence a reduction of the coordination numbers. For the sample with $f_{c}=30 \%, \mathcal{N}_{C}^{C-C}$ at the peak state becomes lowest among various values of $f_{c}$. However, $\mathcal{N}_{C}^{C-F}$ remains still highest (about 35 fine particles around a coarse particle), which compensates for the reduction of $\mathcal{N}_{C}^{C-C}$ and allows this sample to have the best shear strength. After the peak state, $\mathcal{N}_{C}^{C-F}$ reduces drastically. The destruction of the primary skeleton constituted by coarse particles and the drastic reduction in the reinforcement of the primary skeleton by fine particles explain why the sample with $f_{c}=30 \%$ shows a marked softening behavior (Figure 3).
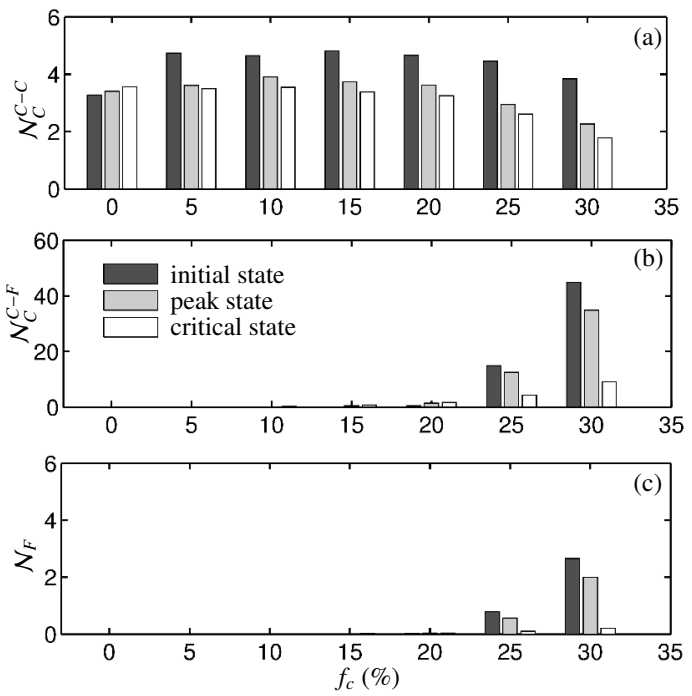

Figure 4. Coordination numbers (a) $\mathcal{N}_{C}^{C-C}$, (b) $\mathcal{N}_{C}^{C-F}$ and (c) $\mathcal{N}_{F}$ versus fine content $f_{c}$ at the initial, peak and critical states.

\subsection{Anisotropy}

One of the particular features of a granular medium is its capability of orienting preferentially its contacts between particles in the major principal stress direction to resist the external deviatoric stress. This feature is called the induced anisotropy, which can be described in terms of the distribution of contact orientation by the so-called fabric tensor $\boldsymbol{H}$. For a gap graded sample for which the particle size is very different from one to another, the particle size should be taken into account in the fabric tensor $\boldsymbol{H}$ :

$$
H_{i j}=\sum_{k} l^{k} n_{i}^{k} n_{j}^{k}
$$

where superscript $k$ runs over all contacts of the sample; $l^{k}$ is the length of the branch vector joining the centers of two particles in contact $k$ and $\boldsymbol{n}^{k}$ is the unit normal vector at contact $k$. For a triaxial loading, $\boldsymbol{H}$ is approximately diagonal and the two transversal components $H_{22}$ and $H_{33}$ are almost equal. In this case, the anisotropy can be quantified by the anisotropy index $H_{d}=\left(H_{11}-H_{33}\right) / \operatorname{Trace}(\boldsymbol{H})$.

Figure 5 shows the evolution of the anisotropy index $H_{d}$ during the triaxial loading for different fine contents $f_{c}$. The effect of $f_{c}$ on the evolution of $H_{d}$ is not significant for $f_{c}<20 \%$ but it appears clearly for $f_{c} \geq 20 \%$. At the peak state, the anisotropy index $H_{d}$ is much higher for $f_{c}=20 \%$ than for $f_{c}<20 \%$. Beyond $f_{c}=20 \%$, the anisotropy at the peak state decreases with $f_{c}$ although the shear strength increases with $f_{c}$ as shown in Figure 3. This is quite surprising. However it can be explained by the fact that at a high fine content, the primary skeleton formed by coarse particles is so well reinforced by fine particles that the granular structure can resist the prescribed loading without resort to induce greatly the anisotropy. It is interesting to note that the softening behavior observed for high fine content (Figure 3 ) is accompanied by a clear decrease of the anisotropy index $H_{d}$ after the peak state.

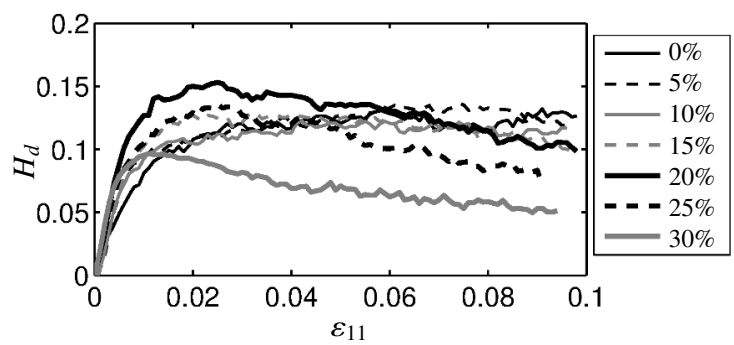

Figure 5. Anisotropy index $H_{d}$ versus axial strain $\varepsilon_{11}$ with different fine contents $f_{c}$.

\subsection{Stress}

Stress applied on the boundary of a granular sample is shared by inter-granular contacts. For a gap-graded sample, three categories of contacts are clearly distinguished. A question that arises here is the participation of each category of contacts in supporting the applied external stress. To answer to this question, we first link the macro-stress tensor $\sigma$ to local information at contacts using the wellknown averaging static operator [10]:

$$
\sigma_{i j}=\frac{1}{V} \sum_{k} f_{i}^{k} l_{j}^{k},
$$

where $V$ is the total volume of the sample; $\boldsymbol{f}^{k}$ et $\boldsymbol{l}^{k}$ are contact force vector and branch vector joining two centers of 
particles in contact. We then decompose stress tensor $\sigma$ into three parts $\sigma^{C-C}, \sigma^{C-F}$ and $\sigma^{F-F}$ which are the contribution of the respective categories of $C-C, C-F$ and $F-F$ contacts. Each part is defined by Equation (3) with superscript $k$ running over the corresponding set of contacts. By doing so, the macroscopic deviator stress $q$ can be decomposed as $q=q^{C-C}+q^{C-F}+q^{F-F}$. The contribution of each category of contacts to $q$ can be calculated from the responding stress tensor, for example the contribution of $C-C$ contacts $q^{C-C}=\sigma_{1}^{C-C}-\sigma_{3}^{C-C}$.
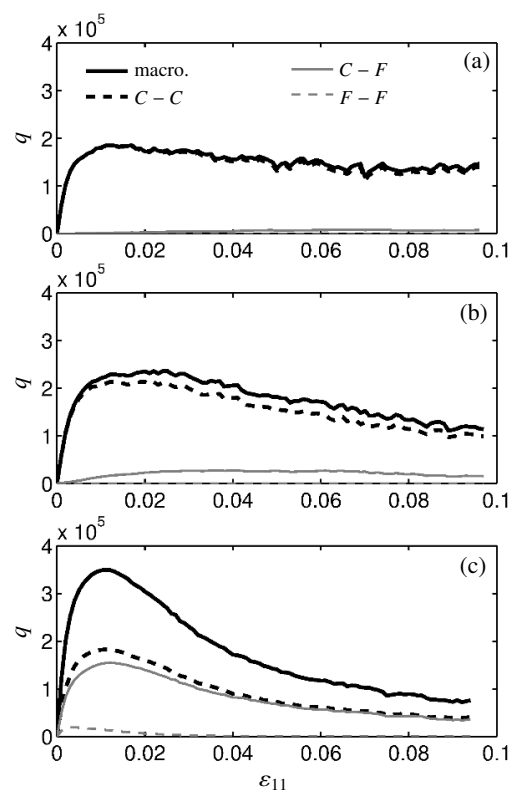

Figure 6. Contribution of each category of contacts $C-C, C-F$ and $F-F$ to the deviator stress $q$ for different fine contents: a) $f_{c}=10 \%$, b) $f_{c}=20 \%$ and c) $f_{c}=30 \%$.

Figure 6 shows the macroscopic deviator stress $q$ and its decomposition $q^{C-C}, q^{C-F}$ and $q^{F-F}$ versus axial strain $\varepsilon_{11}$ for three fine contents $f_{c}=10 \%, 20 \%$ and $30 \%$. It can be seen that, for $f_{c}=10 \%$ and $20 \%$, the applied deviatoric stress is essentially supported by contacts between coarse particles $(C-C)$. However, for $f_{c}=30 \%$, contacts between fine and coarse particles $(C-F)$ participate actively in supporting the applied deviatoric stress. For this high value of fine content, the contribution of $C-F$ contacts is almost equal to that of $C-C$ contacts. The contribution of contacts between fine particles $(F-F)$ is small for the studied range of $f_{c}$. It is interesting to note that $q^{C-C}$ is almost the same whatever the fine content. Combining with the results presented in Section 4.1, we can deduce that the skeleton formed by coarse particles remains almost unchanged and its capability of supporting the external deviatoric stress remains unchanged with increasing fine content. Fine particles which surround coarse particles do not make coarse skeleton stronger. However, $C-F$ contacts take part in supporting the applied deviatoric stress. As shown in Figure 4, as fine content increases, there are more and more fine particles in contact with a coarse particle. As a result, the part of the deviatoric stress supported by $C-F$ contacts increase. This is the reason why the shear strength at high fine content is significantly higher than at low fine content as shown in Figure 3.

\section{Conclusions}

Despite an idealization of granular samples considered in the numerical simulation, this study has brought several insights into the micro-structure of gap-graded samples. For fine content $f_{c}<20 \%$, the skeleton formed by coarse particles is dominant and carries essentially the applied deviatoric stress. As a consequence, fine content does not have a significant effect on the macroscopic behavior. However, for $f_{c} \geq 20 \%$, fine particles come into contact with coarse particles, reinforce the granular skeleton and take part in supporting the external stress, which leads to a higher shear strength. This influence of fine content on soil mechanical behavior highlights the necessity to consider the impact of suffusion process which mobilizes the fine particles and may induce instabilities on hydraulic earth structures.

\section{References}

[1] Y.S. Sail, D. Marot, L. Sibille, A. Alexis, Eur. J. Environ. Civ. Eng. 15, 799 (2011)

[2] R. Salgado, P. Bandini, A. Karim, J. Geotech. Geoenviron. Eng., ASCE 126, 451 (2000)

[3] T.K. Nguyen, N. Benahmed, P.Y. Hicher, M. Nicolas, in Bifurcation and Degradation of Geomaterials in the New Millennium, edited by K.T. Chau, J. Zhao (2015), pp. 113-120

[4] S. Thevanayagam, T. Shenthan, S. Mohan, J. Liang, J. Geotech. Geoenviron. Eng., ASCE 128, 849 (2002)

[5] S̃milauer et al., The Yade Project. DOI 10.5281/zenodo.34073 (http://yade-dem.org/doc/) (2015)

[6] D. Chang, L. Zhang, Soils Found. 53, 569 (2013)

[7] P. Lade, C. Liggio, J. Yamamuro, Geotech. Test. J. 21, 336 (1998)

[8] N. Minh, Y. Cheng, Géotechnique 63, 44 (2013)

[9] T. Shire, C. O'Sullivan, K. Hanley, in Geomechanics from Micro to Macro, edited by K. Soga, K. Kumar, G. Biscontin, M. Kuo (2014), pp. 231-236

[10] J. Christoffersen, M. Mehrabadi, S. Nemat-Nasser, J. Appl. Mech. 48, 339 (1981) 